\title{
PERSISTENCIA DE ANTICUERPOS MATERNALES CONTRA CISTICERCOSIS PORCINA Y SU EFECTO EN LA INTERPRETACIÓN DE RESULTADOS DEL EITB
}

\author{
Alberto Ccama S. ${ }^{1}$, Armando González Z. ${ }^{2,3}$, Néstor Falcón P. ${ }^{2}$ y Teresa Bernal R. ${ }^{2}$
}

\section{AbSTRACT}

The study evaluate the effect of maternal antibodies against porcine cysticercosis on the interpretation of EITB test results. This data set was obtained from epidemiologic studies developed at Quilcas district, Huancayo, during a period of 4 years (1995-1998). Samples from 298 piglets and their respective dams were analized; animals were stratified by sex and immune status. Maternal antibodies persisted in the blood stream until piglets were 148.8 days old (117-242), and without significant differences between sexes. The average age in which positive piglets to EITB got infected was 170, whereas negative piglets got infected at 179.3 days of age (98-261) and without statistical differences due to sex. The immune status of piglets and dams did not interfere with age of infection. The Cox Regression showed that none of the variables under evaluation constituted a risk factor for the infection with cysticercus. This study demonstrated that maternal antibodies against porcine cysticercosis can interfere in the interpretation of results of epidemiologic studies when piglets younger than 8 month of age are sampled, probably due to the fact that the EITB test does not discriminate between material antibodies than those selfacquired as result of a natural infection.

Key words: maternal antibodies, porcine cysticercosis, EITB

\section{Resumen}

EI presente estudio evaluó el efecto de la persistencia de anticuerpos maternales contra cisticercosis porcina en la interpretación de los resultados de la prueba de EITB. Se utilizó una base de datos pre-existente con resultados de 4 años de estudios epidemiológicos (1995-1998) en el distrito de Quilcas, Huancayo. Se analizaron los resultados de 298 crías, clasificadas según sexo y estado inmunológico de las crías y sus madres. EI tiempo promedio de persistencia de anticuerpos maternales que fue de 148.8 días de edad (117- 242) sin diferencias estadísticas entre sexos. EI tiempo promedio en que los lechones positivos a EITB se infectaron fue de 170 días de edad, en tanto que los lechones negativos se infectaron a los 179.3 días de edad (98-261), sin diferencias estadísticas entre sexos. No hubo diferencias significativas entre el tiempo de infección según el estado inmunológico de las crías o de las madres (positivas o negativas). La Regresión de Cox mostró que ninguna de las variables bajo estudio representó un factor de riesgo en el tiempo de infección con cisticercos. El estudio determinó que los

${ }^{1}$ Laboratorio de Microbiología e Inmunología, FMVZ-UNA, Puno

${ }^{2}$ Laboratorio de Medicina Veterinaria Preventiva, FMV-UNMSM

${ }^{3}$ E-mail: agonzalesz@vet.unmsm.edu.pe 
anticuerpos maternales contra cisticercosis pueden interferir en la interpretación de los resultados de investigaciones epidemiológicas de campo cuando las muestras provienen de lechones de menos de 8 meses de edad, debido a que la prueba de EITB no discrimina anticuerpos maternales de los formados por una infección natural.

Palabras clave: anticuerpos maternales, cisticercosis porcina, EITB

\section{INTRODUCCIÓN}

La cisticercosis porcina es una enfermedad parasitaria causada por el Cysticercus cellulosae, el estadío larvario de la Taenia solium. EI hombre es el único hospedero definitivo de esta tenia y tiene como hospederos intermediarios al cerdo doméstico y accidentalmente al mismo ser humano (Acha y Szyfres, 1986; Borchert, 1981; SartiGutiérrez et al., 1992; Soulsby, 1987). Las larvas de Taenia solium usualmente sobreviven en los tejidos de porcinos y humanos sin ocasionar procesos inflamatorios, a pesar que pueden tener anticuerpos circulantes, debido a que los cisticercos han logrado mecanismos de evasión y modulación inmune (Evans y The Cysticercosis Working Group in Peru, 1999).

Los factores de riesgo más importantes en áreas rurales están referidos a la crianza doméstica de cerdos sin confinamiento, a la falta de hábitos de higiene personal, a la falta de letrinas u otro sistema de disposición de excretas humanas que permiten que los cerdos tengan acceso a excrementos humanos, y a un deficiente sistema de referencia a niveles de atención médica especializada (OPS, 1993). La cisticercosis representa un problema de salud pública debido a que produce una grave patología en el hombre; además constituye un serio problema económico debido a las pérdidas que significa el decomiso de las carcasas de los animales parasitados (Loo y Braude, 1982; Mathias et al., 1983; Damonte, 1983).

Los campesinos sudamericanos diagnostican la cisticercosis mediante la prueba de la lengua. Ésta se realiza palpando la cara inferior y las superficies laterales de la lengua, al lado del frenillo (González et al., 1993; Borchert, 1981), pudiendo llegar a alcanzar una sensibilidad de $70 \%$ y una especificidad del $100 \%$ cuando el examen es realizado por personas con experiencia en el método (González et al., 1990). Por otro lado, los cisticercos se pueden observar en los músculos de la cruz y de la región lumbar en animales beneficiados (Acha y Szyfres, 1986; Borchert, 1981).

La técnica diagnóstica de laboratorio más utilizada en la última década es la prueba de Western Blot (EITB) (Tsang et al., 1989; Tsang et al., 1991). Está considerada como la mejor herramienta de diagnóstico para cisticercosis humana y porcina (Flisser et al., 1990; García et al., 1991; Pathak et al., 1994; Schantz et al., 1994; González et al., 1999) ya que alcanza una sensibilidad del $98 \%$ y una especificidad del $100 \%$. Además, tiene la ventaja de no presentar reacciones cruzadas con otros parásitos (Tsang et al., 1989; Tsang et al., 1991).

En base a la prueba de EITB se están realizando los últimos trabajos de investigación sobre la epidemiología de cisticercosis porcina a nivel mundial. En un estudio preliminar sobre inmunidad pasiva realizado por Barrón (1996) con lechones de una marrana infectada con cisticercos proveniente de una zona endémica (Huancayo) se encontró que los anticuerpos maternales permanecen detectables a la prueba de EITB hasta aproximadamente los 8 meses de edad. Tomando esta referencia, el objetivo del estudio fue determinar el efecto que causan los anticuerpos maternales contra cisticercosis porcina en la interpretación de los resultados de la prueba de EITB en trabajos epidemiológicos de campo. 


\section{Materiales y Mí́todos}

\section{Lugar de estudio}

EI análisis de la información se realizó en el Laboratorio de Medicina Veterinaria Preventiva de la Facultad de Medicina Veterinaria de la Universidad Nacional Mayor de San Marcos. Se utilizó una base de datos conteniendo los resultados de EITB para cisticercosis porcina obtenidos en un periodo de cuatro años (1995-1998). EI muestreo inicial de sangre para la prueba de EITB se realizó en el distrito de Quilcas, provincia de Huancayo, departamento de Junín; zona donde la cisticercosis porcina alcanza niveles de prevalencia de $72 \pm 4 \%$ por la que es considerada hiperendémica (Bernal, 1996).

\section{Base de datos y muestreo}

La base de datos contiene los resultados de EITB para cisticercosis de 5,291 porcinos criollos, pertenecientes a los comuneros del distrito de Quilcas, quienes alimentan sus porcinos con residuos de cosecha y de cocina. El manejo de los animales era de tipo semi-intensivo, ya que a veces los mantienen amarrados a estacas cerca de las viviendas y otras veces los dejan libres en sus chacras alimentándose con residuos de cosecha. EI desconocimiento del ciclo biológico de la cisticercosis por parte de los comuneros no les ha permitido controlar la enfermedad.

EI muestreo fue de intención o razonado, seleccionando de la base de datos a 298 lechones con dos o más resultados de EITB para cisticercosis (los muestreos de sangre para la prueba de EITB se realizaron cada dos meses) y donde la primera muestra se tomó después del primer mes de vida de la cría. Los animales fueron agrupados, con fines de análisis, por sexo (174 hembras y 124 machos) y por estado inmunológico de las crías y sus madres (positivos o negativos). Se trabajó con camadas completas, con un promedio de 3 lechones por camada.

\section{Análisis de datos}

Se determinó el tiempo en el que las crías positivas a EITB para cisticercosis pasaban a ser negativas o incrementaban el número de bandas reactivas a la prueba, y el tiempo en que las crías negativas pasaban a ser positivas a la prueba. Así mismo, se determinó la media y los limites superior e inferior del tiempo de persistencia de los anticuerpos.

Para evaluar el tiempo de ocurrencia de la infección de las crías se utilizó la curva de Kaplan Meier, considerando la condición inmunológica de las madres. Además, se evaluó el efecto de la variable sexo sobre el tiempo de infección en los lechones, mediante la prueba de Regresión de Cox.

\section{Resultados}

Se obtuvo una muestra de 298 crías que reunían las características de interés para el desarrollo del presente estudio. De allí, 44 crías negativas eran descendientes de madres negativas y 254 crías (91 positivas y 163 negativas) eran descendientes de madres positivas al EITB para cisticercosis.

De las 91 crías positivas, 49 presentaron el mismo número de bandas reactivas al EITB que la madre. Se hizo el seguimiento en los resultados de la prueba de EITB de estos lechones hasta que las bandas reactivas desaparecieran. EI tiempo promedio de persistencia de los anticuerpos maternales para este grupo fue de 148.8 días de edad (117-242). No se hallaron diferencias estadísticamente significativas entre los tiempos de infección según sexo (146 días de edad en machos y 155 días en hembras).

Las 42 crías positivas restantes incrementaron el número de sus bandas reactivas al EITB con relación a sus madres, lo que se consideró como una respuesta inmunológica a la infección con cisticercos. Las bandas aumentaron antes o en simultáneo 
a la desaparición de los anticuerpos maternales. La edad promedio en que estos lechones se infectaron con cisticercos fue de 170 días, sin que hubiera diferencias estadísticas entre los tiempos de infección debido a la variable sexo (machos: 178; hembras: 161 días).

La edad promedio en que se infectaron las crías negativas $(\mathrm{n}=207)$ con cisticercos fue de 179.3 días (98-261). No se encontró diferencias estadísticas significativas entre los tiempos de infección debido al sexo ni al estado inmunológico de las crías o de las madres (crías de madres negativas: 178; crías de madres positivas: 174 días).

La evaluación del tiempo de infección de los grupos de crías positivas y negativas al EITB para cisticercosis no reportó diferencias estadísticas significativas. La Regresión de Cox mostró que ninguna de las variables bajo estudio representa un factor de riesgo en el tiempo de infección con cisticercos.

\section{Discusión}

EI estudio demostró que los anticuerpos maternales contra la cisticercosis porcina pueden interferir en la interpretación de resultados de la prueba de EITB en estudios epidemiológicos de campo, cuando se realiza un solo muestreo en crías menores de 8 meses de edad. Esto se debe a que la prueba detecta anticuerpos, pero no discrimina entre aquellos producidos por una infección natural y los anticuerpos transmitidos por la madre mediante el calostro; pudiendo de esta manera sobredimensionarse los verdaderos niveles de infección. Esto podría superarse si se evalúa el comportamiento inmunológico de las crías en muestreos consecutivos con un intervalo de un mes, considerando que la producción de anticuerpos como respuesta a una infección es después de 10 a 15 días (Tizard, 1998).

EI análisis de los resultados muestra que todas las crías, independientemente de la edad y sexo, están en peligro de infectarse con cisticercos aún si descienden de madres positivas a EITB para cisticercosis. Esto está indicando que los anticuerpos maternales no son protección suficiente contra las infecciones, o que los cisticercos pueden sobrevivir a la acción de los componentes del sistema inmunológico del porcino, evadiendo o modulando su respuesta (Evans y The Cysticersosis Working Group in Peru, 1999).

La mayor presencia de crías negativas descendientes de madres positivas fue un hallazgo no esperado. Esto podría explicarse por fallas en la producción de calostro por la madre, fallas en la ingestión de calostro o alteraciones en la absorción de los anticuerpos por las crías (Tizard, 1998).

\section{Conclusiones}

La presencia de anticuerpos maternales contra cisticercosis porcina interfiere directamente en la interpretación de los resultados de la prueba de EITB cuando se realiza un muestreo único, al no discriminar los anticuerpos producidos por infección, de los transferidos mediante el calostro.

\section{Literatura Citada}

1. Acha, P.; B. Szyfres. 1986. Zoonosis y enfermedades transmisibles comunes al hombre y los animales. $2^{\text {da }}$ ed. p 763-774. OPS. Washington, EE.UU.

2. Barrón, E. 1996. Persistencia de anticuerpos maternales en crías de una marrana infectada con Cysticercus cellulosae. Tesis de Médico Veterinario. Facultad de Medicina Veterinaria, Univ. Nacional Mayor de San Marcos. Lima. $33 \mathrm{p}$.

3. Bernal, T. 1996. Evaluación de la cisticercosis porcina en el distrito de Quilcas, Huancayo. Tesis de Médico Veterinario. Facultad de Medicina Veterinaria, Univ. Nacional Mayor de San Marcos. Lima. $45 \mathrm{p}$. 
4. Borchert, A. 1981. Parasitología Veterinaria. $3^{\text {ra }}$ ed. p 162-166. Ed. Acribia. Zaragoza, España.

5. Damonte, L.J. 1983. Desconocimiento de la epidemiología de la cisticercosis en México. Sal. Pub. Mex. 25: 301-305.

6. Evans, C.; The Cysticercosis Working Group in Peru. 1999. The immunology of the host-parasite relationship in Taenia solium cysticercosis: Implications for prevention and therapy. En: García, H; Martínez, S. (eds). Taenia solium Taeniasis / Cysticercosis. $2^{\text {nd }}$ ed. p 2535. Ed. Universo. Lima.

7. Flisser, A.; A. Plancarte; D. Correa; E. Rodríguez del Rosal; M. Feldman; M. Sandoval. 1990. New approaches in the diagnosis of Taenia solium cysticercosis and teniasis. Ann. Parasitol. Hum. Comp. 65 (Suppl. 1): 95-98.

8. García, H.H.; M. Martínez; R.H. Gilman; G. Herrera; V.C. Tsang. 1991. Diagnosis of cysticercosis in endemic regions. Lancet 338: 549-551.

9. González, A.E.; R.H. Ccama; R. Gilman; V.C. Tsang. 1990. Prevalence and comparison of serological assay, necropsy, and tongue examination for the diagnosis of porcine cysticerosis in Peru. Am. J. Trop. Med. Hyg. 43: 194-199.

10. González, A.E.; M. Castro; R.H. Gilman. 1993. The marketing of cysticercosis pigs in the Sierra of Peru. Bull. Health Organ. 71: 223-228.

11. González, A.E.; C. Gavidia; N. Falcón; C. Evans. 1999. Porcine cysticercosis: epidemiology, diagnosis and treatment. En: García, H.; Martínez, S. (eds). Taenia solium Taeniasis/Cysticercosis. $2^{\text {dd }}$ ed. p 97-155. Ed. Universo. Lima.

12. Loo, L; A. Braude. 1982. Cerebral cysticercosis in San Diego: a report of 23 cases and a review of the literature. Medicine 61: 341.

13. Mathias, Z.; Z. Pawlowski; E.J.L. Soulsby. 1983. Guidelines for surveillance, prevention and control of cysticercosis/taeniasis. p 71-72. World
Health Organization. Geneva, Switzerland (WHONPH 83.49).

14. OPS. 1993. Desarrollo y fortalecimiento de los sistemas locales de Salud. HBSSilo 23: 63. EE.UU.

15. Pathak, K.M.; J.C. Allan; K. Ersfeld; P.S. Craig. 1994. A Western Blot and ELISA assay for the diagnosis of Taenia solium infection in pig. Vet. Parasitol. 53: 209-217.

16. Sarti-Gutiérrez, E.; P. Schantz; A. Plancarte; M. Wilson; I. Gutiérrez; A. López; J. Roberts; A. Fusser. 1992. Prevalence and risk factors for Taenia solium taeniasis and cysticercosis in humans and pig in a village in Morelos, Mexico. Am. J. Trop. Med. Hyg. 46: 677685.

17. Schantz, P.M.; E. Sarti; A. Plancarte; M. Wilson; J.L. Criales; J. Roberts; A. Flisser. 1994. Community-based epidemiological investigations of cysticercosis due to Taenia solium: Comparison of serological screening test and clinical findings in two populations in Mexico. Clin. Infec. Dis. 18: 879-885.

18. Soulsby, E.J. 1987. Parasitología y enfermedades parasitarias en los animales domésticos. $7^{\text {ma }}$ ed. p 106-112. Ed. Interamericana. México.

19. Tizard, I. 1998. Inmunología Veterinaria. $5^{\text {ta }}$ ed. Mc Graw-Hill Interamericana. México.

20. Tsang, V.C.; J.A. Brand; A.E. Boyer. 1989. An Enzime-linked immunoelectrotransfer Blot Assay and glycoprotein antigen for diagnosing human cysticercosis (Taenia solium) J. Infect. Dis. 159: 50-59.

21. Tsang, V.C.; J.A. Pilcher; W. Zhou; A.E. Boyer; E.I. Kamango-Sollo; M.L. Rhoads. 1991. Efficacy of the inmunoblot assay for cysticercosis in pigs and modulated expression of distinct IgM/ IgG activities to Taenia solium antigens in experimental infections. Vet. Inmunol. Immunopathol. 29: 69-78. 